\title{
Antifungal stewardship in a tertiary care paediatric hospital: the PROAFUNGI study
}

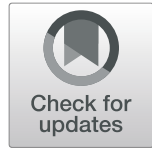

Natalia Mendoza-Palomar ${ }^{1 \dagger}$, Beatriz Garcia-Palop ${ }^{2 \dagger}$, Susana Melendo ${ }^{1}$, Maria Teresa Martín ${ }^{3}$, Berta Renedo-Miró2 Pere Soler-Palacin $^{1 *}$ and Aurora Fernández-Polo ${ }^{2}$

\begin{abstract}
Background: The increasing use of antifungal drugs (AF) in children and the concern for related adverse events and costs has led to the development of specific AF stewardship programmes (AFS). Studies in adult patients have shown improvements in AF prescription and usage after implementation, but paediatric data are scant. The aim of this PROAFUNGI study was to describe the use and appropriateness of AF in a high complexity paediatric centre.

Methods: Observational, prospective, single-centre, modified point-prevalence study (11 surveys, July-October 2018), including paediatric (<18 years) patients receiving at least one systemic AF. Prescriptions were evaluated by the AFS team.

Results: The study included 119 prescriptions in 55 patients (53\% males, median age 8.7 years [IQR 2.4-13.8]). The main underlying condition was cancer (45.5\% of patients; HSCT in $60 \%$ of them); and the first indication for AF was prophylaxis (75 prescriptions, 63.2\%). Liposomal amphotericin B was used most commonly (46\% prescriptions), mainly as prophylaxis (75\%). Among the 219 evaluations, 195 (89\%) were considered optimal. The reason for nonoptimal prescriptions was mostly lack of indication (14/24), especially in critical patients with ventricular assist devices. The use of AF without paediatric approval accounted for 8/24 inappropriate prescriptions.

Conclusions: A high rate of AF appropriateness was found for the children's hospital as a whole, in relation with a well-established AFS. Nonetheless, the identification of specific areas of improvement should guide future actions of the AFS team, which will focus mainly on prophylaxis in critically ill patients receiving circulatory assistance and the use of non-approved drugs in children.
\end{abstract}

Keywords: Antifungal therapy, Paediatrics, Antimicrobial management

\footnotetext{
*Correspondence: psoler@vhebron.net

${ }^{+}$Natalia Mendoza-Palomar and Beatriz Garcia-Palop contributed equally to this work.

${ }^{1}$ Pediatric Infectious Diseases and Inmunodeficiencies Unit, Hospital Universitari Vall d'Hebron, Institut de Recerca Vall d'Hebron, Universitat Autònoma de Barcelona, Passeig de la Vall d'Hebron, 119-129, 08035 Barcelona, Spain

Full list of author information is available at the end of the article
}

(C) The Author(s). 2021 Open Access This article is licensed under a Creative Commons Attribution 4.0 International License, which permits use, sharing, adaptation, distribution and reproduction in any medium or format, as long as you give appropriate credit to the original author(s) and the source, provide a link to the Creative Commons licence, and indicate if changes were made. The images or other third party material in this article are included in the article's Creative Commons licence, unless indicated otherwise in a credit line to the material. If material is not included in the article's Creative Commons licence and your intended use is not permitted by statutory regulation or exceeds the permitted use, you will need to obtain permission directly from the copyright holder. To view a copy of this licence, visit http://creativecommons.org/licenses/by/4.0/ The Creative Commons Public Domain Dedication waiver (http://creativecommons.org/publicdomain/zero/1.0/) applies to the data made available in this article, unless otherwise stated in a credit line to the data. 


\section{Background}

In recent years, the increase in the number of immunosuppressed children at risk of invasive fungal infection (IFI) has led to a higher use of both prophylactic and therapeutic antifungal drugs (AF) in this population [1]. In these patients, AF prescription may be challenging due to unspecific clinical presentation, lower performance of diagnostic tests, variable pharmacokinetics related to maturation changes and lack of clinical trials in children [1-3].

Unnecessary AF use entails substantial risk of toxicity and interactions, along with higher drug resistances and significant costs [3]. In the last 5 years, AF drugs accounted for around one-third of antimicrobial usage (13.79\% of all days of therapy [DOT] per 100 patientdays [PD]) but $75 \%(€ 638,403)$ of total annual antiinfective expenditure in our hospital (unpublished data).

In response to this concern, specific AF stewardship programmes (AFS) are being developed to improve the clinical outcome of patients receiving AF drugs and to ensure optimal prescription in terms of spectrum, dose, therapeutic drug monitoring, duration and route of administration while avoiding adverse effects and unnecessary costs [3]. Interventions are extrapolated from the antibiotic stewardship programmes and include audits with feedback, guideline and protocol development, educational programmes and prescription evaluation $[4,5]$. Although AFS are relatively new, a systematic review in 2017 identified 14 papers describing AFS in adult patients and the improvements in AF prescription and use after implementation [6]. In contrast, data about paediatric AFS are scant and mainly limited to adult programmes including children or to specific situations such as invasive Candida spp. infection or cancer [7, 8].

The aim of this PROAFUNGI study was to describe the use and appropriateness of AF in a high complexity paediatric centre.

\section{Methods}

The children's hospital at Vall d'Hebron Barcelona Hospital Campus is a reference tertiary care centre with a national reference programme of solid organ transplantation (SOT) and hematopoietic stem cell transplantation (HSCT) with 35 and 40 yearly transplantations, respectively, as well as a 20-bed paediatric ICU (PICU), a 44bed neonatal ICU (NICU) and a large surgery programme with around 2700 inpatient procedures per year. The institutional paediatric antimicrobial stewardship program (PROA-NEN) was formally founded in 2015 and aims to improve clinical outcomes, reduce antimicrobial-related adverse events and ensure the cost-effective use of treatments. During 2018, a specific AFS team was created, involving 3 pharmacists, 3 paediatric infectious diseases specialists and 1 microbiologist.
The PROAFUNGI study is an observational, prospective, single-centre, modified point-prevalence survey (mPPS) study performed from July to October 2018. Admitted patients receiving AF were identified through the electronic prescription system (Silicon ${ }^{\circ}$ v11, Grifols International, S.A., Sant Cugat del Vallès, Spain and Centricity ${ }^{\mathrm{Ts}}$ Critical Care 8.1 SP7, General Electric Company, Barrington, United States) and data were collected weekly. All consecutive paediatric (< 18 years) patients receiving at least one systemic AF were included. Patients were included again if a new AF course was started. Additionally, one AF prescription was recorded for each week in which the drug was prescribed. Patients receiving AF for less than 48 $\mathrm{h}$ were excluded.

Demographic, clinical and treatment data were obtained from the patient's electronic medical record (SAP $\odot$ NetWeaver 7.0 SPS37, California, United States), including age, sex, underlying disease, AF indication and IFI category. The patient's IFI was classified as possible, probable or proven according to the European Organization for Research and Treatment of Cancer/Mycoses Study Group (EORTC/MSG) definitions [9]. Additionally, patients at risk of IFI and or with clinical suspicion of IFI were added as other categories to include situations not listed in the EORTC/ MSG criteria. The AF indication was classified as prophylaxis (primary or secondary) or treatment (empirical, pre-emptive or targeted). Pharmacological data (AF, route of administration, dose and frequency) were collected from the abovementioned electronic prescription systems.

After the collection, data were evaluated by two independent members of the AFS team. In case of disagreement, a third member was consulted to achieve consensus. Following the guidelines for the implementation of antimicrobial stewardship programs in Spanish hospitals, optimal prescription was defined as "necessary" (if the clinical situation required the use of an AF, either prophylactic and therapeutic), "appropriate" (if the drug was active against the causative microorganism and adjusted to the patient's characteristics), and "adequate" (if the dose, duration and route of administration were correct, while in concordance with local protocols, or international guidelines if local protocols were not available) [10].

Qualitative variables were described as the number and percentage, and quantitative variables as the mean \pm standard deviation (SD) or median (IQR). The statistical analysis was carried out with Microsoft $^{\ominus}$ Office $^{\text {Excel }}{ }^{\odot}$ 2007 (12.0.4518.1014).

The local Ethics Committee for Clinical Research approved the study in March 2018 (code VAL-ANT2017-01). 


\section{Results}

\section{Patient and fungal infection characteristics}

During the study period, 55 patients were included; $53 \%$ were males and median age was 8.7 years (IQR 2.4-13.8 years). The main underlying condition was cancer (25, $45.5 \% ; 15$ [60\%] of them having undergone HSCT); and the most common situation leading to AF prescription was patients at risk of IFI (27; 49\%). Detailed data are provided in Table 1.

\section{Antifungal prescription characteristics}

A total of 119 AF prescriptions were analysed. The indication was prophylaxis in $75(63.2 \%)$ cases (70 primary and 5 secondary) and treatment in 44 (36.8\%): 30 (25\%) empirical; 1 (0.8\%) pre-emptive; 13 (11\%) targeted. Drug distribution by type of patient and indication is detailed in Table 2. Most drugs (93.3\%) were prescribed as monotherapy (52 patients), while dual and triple therapy were only used in 1 patient (2 prescriptions, 1.7\%) and 2 patients (6 prescriptions, 5\%), respectively.

\section{Prescription quality assessment}

During the study period, the PROAFUNGI working group performed a total of 11 surveys, including 219 prescription evaluations. From the 219 evaluated

Table 1 Patient Characteristics

\begin{tabular}{|c|c|}
\hline & Total \\
\hline Clinical characteristics, $n$ & 55 \\
\hline - Age, y, median (IQR) & $8.7(2.4-13.8)$ \\
\hline - Male sex, $n(\%)$ & $29(53)$ \\
\hline \multicolumn{2}{|l|}{ Underlying disease } \\
\hline - Cancer, n (\%) & $25(45.5)$ \\
\hline • HSCT, n (\%) & $15 / 25(60)$ \\
\hline - Critical paediatric patients, n (\%) & $14(25.5)$ \\
\hline - Critical newborns, $n$ (\%) & $5(9)$ \\
\hline - Primary immunodeficiencies, $n$ (\%) & $5(9)$ \\
\hline - Solid organ transplant, $n$ (\%) & $3(5.5)$ \\
\hline - Other, $n(\%)$ & $3(5.5)$ \\
\hline \multicolumn{2}{|l|}{ Clinical situation $^{a}$} \\
\hline • Risk of IFI, n (\%) & $27(49)$ \\
\hline • Suspicion of IFI, n (\%) & $6(11)$ \\
\hline • Possible IFI, n (\%) & $14(25)$ \\
\hline - Probable IFI, n (\%) & $2(4)$ \\
\hline • Proven IFI, n (\%) & $6(11)$ \\
\hline \multicolumn{2}{|l|}{ Microorganisms } \\
\hline - Candida spp. & $2 / 6$ \\
\hline - Moulds & $4 / 6$ \\
\hline
\end{tabular}

Abbreviations: IFI invasive fungal infection

a IFI category was defined according to the European Organization for Research and Treatment of Cancer/Mycoses Study Group (EORTC/MSG) definitions prescriptions, 24 (11\%) were considered non-optimal. As shown in Table 2, the main reason for a non-optimal evaluation was lack of indication (14/24), followed by disagreement with the local protocols (12/24), mainly in heart-transplanted and HSCT patients. Institutional protocols were available for $117 / 144$ (81\%) prophylactic and 40/75 (53\%) treatment prescriptions. Moreover, the departments with the highest AF use (cancer department and PICU) issued most non-optimal prescriptions (16.7 and 58.3\%, respectively).

Liposomal amphotericin B (LAMB) was used commonly and was also the main drug considered non-optimal (Table 3), essentially due to non-optimal prescriptions related to prophylaxis in patients receiving circulatory assistance (7/12): 2 patients with ventricular assist device (VAD) who had an unnecessary prescription and 1 receiving extracorporeal membrane oxygenation (ECMO) support who had an inappropriate prescription due to an excessive dosage.

Two non-approved drugs in paediatrics at the time of the study, isavuconazole (5 prescriptions in one patient) and anidulafungin (3 prescriptions in one patient), accounted for a significant number of non-optimal prescriptions due to the availability of alternative approved drugs as empirical treatment in one patient with HSCT and acute renal impairment who could have been treated with oral voriconazole and one patient with portal cavernomatosis who could have been treated with micafungin, respectively.

\section{Discussion}

The present study demonstrates a significantly high percentage of optimal prescription of AF in children at a referral hospital but has also detected some points of improvement mainly related to prophylaxis in critically ill patients receiving circulatory assistance and the use of non-approved drugs in children.

According to previous published articles, cancer patients were those receiving AF more frequently $[8,11$, 12]. In a retrospective cohort study conducted in 25 paediatric hospitals in the USA, patients with malignancies represented $42 \%$ of overall AF use [11]. In our cohort, a large proportion of AF prescriptions were ordered for HSCT patients (15 patients, accounting for 43 prescriptions, $36 \%$ of all AF prescriptions and $67 \%$ of cancer patient prescriptions), as our centre is a national referral transplant unit. Similarly, in a study including only children with cancer, HSCT patients accounted for $78 \%$ of overall AF prescriptions [8]. Regarding patients' age, the Antibiotic Resistance and Prescribing in European Children (ARPEC) study reported higher AF use in paediatric patients (83\%) compared with newborns (17\%), as did another survey including more than 50 hospitals in the USA (76 DOT/1000 patient-days in paediatric patients compared with 14 DOT/1000 
Table 2 Antifungal Prescription Characteristics and Adequacy

\begin{tabular}{|c|c|c|c|c|}
\hline & & Total & Prophylaxis & Treatment \\
\hline Antifungal pre & ons, $n$ & 119 & 75 & 44 \\
\hline Indication for $\mathrm{A}$ & & & & \\
\hline Primary prop & $n(\%)$ & $70(58.8)$ & $70(93.3)$ & \\
\hline Secondary pr & xis, n (\%) & $5(4.4)$ & $5(6.7)$ & \\
\hline Empirical the & (\%) & $30(25)$ & & $30(68.2)$ \\
\hline Pre-emptive &,$n(\%)$ & $1(0.8)$ & & $1(2.3)$ \\
\hline Targeted the & (\%) & $13(11)$ & & $13(29.5)$ \\
\hline Underlying dise & & & & \\
\hline Cancer, $n(\%)$ & & $64(54)$ & $48(64)$ & $16(36.4)$ \\
\hline$H S C T, n(\%)$ & & $43 / 64(67)$ & $35 / 48(73)$ & $8 / 16(50)$ \\
\hline Critical paedi & atients, $n$ (\%) & $29(24)$ & $16(21.4)$ & $13(29.5)$ \\
\hline Critical newb & (\%) & $7(6)$ & $3(4)$ & $4(9.1)$ \\
\hline Primary imm & iciencies, $n$ (\%) & $11(9)$ & $7(9.3)$ & $4(9.1)$ \\
\hline Solid organ $t$ & int, n (\%) & $3(3)$ & $0(0)$ & $3(6.8)$ \\
\hline Other, $n(\%)$ & & $5(4)$ & $1(1.3)$ & $4(9.1)$ \\
\hline AF agent & & & & \\
\hline Liposomal ar & ricin B, n (\%) & $55(46.2)$ & $41(54.6)$ & $14(32)$ \\
\hline Posaconazole & & $21(17.7)$ & $21(28)$ & $0(0)$ \\
\hline Anidulafungi & & $13(10.9)$ & $3(4)$ & $10(23)$ \\
\hline Fluconazole, & & $12(10.1)$ & $6(8)$ & $6(14)$ \\
\hline Micafungin, I & & $6(5.0)$ & $2(2.7)$ & $4(9)$ \\
\hline Isavuconazol & & $5(4.2)$ & $0(0)$ & $5(11)$ \\
\hline Voriconazole & & $4(3.4)$ & $0(0)$ & $4(9)$ \\
\hline Itraconazole, & & $2(1.7)$ & $2(2.7)$ & $0(0)$ \\
\hline Caspofungin & & $1(0.8)$ & $0(0)$ & $1(2)$ \\
\hline Antifungal pre & on evaluations, $n$ & 219 & 144 & 75 \\
\hline Non-optimal & ptions, n (\%) & $24(11)$ & $12(8)$ & $12(16)$ \\
\hline Unnecessary, & & $14(6)$ & $10(7)$ & $4(5)$ \\
\hline Inappropriate & & $2(1)$ & $0(0)$ & $2(3)$ \\
\hline Inadequate & Incorrect dose, $n(\%)$ & $7(3)$ & $4(3)$ & $3(4)$ \\
\hline & Incorrect route of administration, $n$ (\%) & $0(0)$ & $0(0)$ & $0(0)$ \\
\hline & Incorrect duration, $n$ (\%) & $7(3)$ & $7(5)$ & $0(0)$ \\
\hline & Disagreement with institutional protocol, when available, $n(\%)$ & $12(8)^{a}$ & $4(3)^{a}$ & $8(20)^{a}$ \\
\hline Non-optimal pr & on distribution among underlying disease, $n$ (\%) & & & \\
\hline Cancer & & $4(16.7)$ & $2(16.7)$ & $2(16.7)$ \\
\hline$H S C T, n(\%)$ & & $4 / 4(100)$ & $2 / 2(100)$ & $2 / 2(100)$ \\
\hline Critical paedi & atients, $n(\%)$ & $14(58.3)$ & $8(66.7)$ & $6(50)$ \\
\hline Critical newb & (\%) & $1(4.2)$ & $1(8.3)$ & $0(0)$ \\
\hline Primary imm & iciencies, $n$ (\%) & $1(4.2)$ & $1(8.3)$ & $0(0)$ \\
\hline Solid organ t & int, $n(\%)$ & $1(4.2)$ & $0(0)$ & $1(8.3)$ \\
\hline Other, $n(\%)$ & & $3(12.5)$ & $0(0)$ & $3(25)$ \\
\hline
\end{tabular}

Abbreviations: $A F$ antifungal

${ }^{\text {a }}$ Calculated as a percentage of the prescriptions for which a local institutional protocol was available: 157 total, 117 prophylaxis, 40 treatment 
Table 3 Assessment of Prescription Quality According to Drug

\begin{tabular}{|c|c|c|c|c|}
\hline Drug & $\begin{array}{l}\text { Patients, } \\
n\end{array}$ & $\begin{array}{l}\text { Prescriptions } \\
\text { Evaluated, } n\end{array}$ & $\begin{array}{l}\text { Non-optimal Prescriptions } \\
(n, \%)\end{array}$ & Cause $^{a}$ \\
\hline \multirow{3}{*}{$\begin{array}{l}\text { Liposomal } \\
\text { amphotericin B }\end{array}$} & \multirow[t]{3}{*}{27} & \multirow[t]{3}{*}{105} & \multirow[t]{3}{*}{$12(11 \%)$} & - Unnecessary (9) \\
\hline & & & & - Inappropriate (2) \\
\hline & & & & $\begin{array}{l}\text { - Inadequate: incorrect dose (5), incorrect duration (7) } \\
\text { disagreement with institutional protocol }{ }^{\mathrm{b}}(5)\end{array}$ \\
\hline Posaconazole & 12 & 32 & $1(3 \%)$ & - Inadequate, incorrect dose (1) \\
\hline Anidulafungin & 11 & 29 & $3(10 \%)$ & - Unnecessary (3) \\
\hline Fluconazole & 11 & 25 & $1(4 \%)$ & - Inadequate, incorrect dose (1) \\
\hline Isavuconazole & 2 & 9 & $5(56 \%)$ & 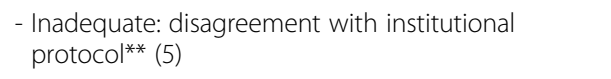 \\
\hline \multirow[t]{2}{*}{ Micafungin } & \multirow[t]{2}{*}{3} & \multirow[t]{2}{*}{7} & \multirow[t]{2}{*}{$2(29 \%)$} & - Unnecessary (2) \\
\hline & & & & $\begin{array}{l}\text { - Inadequate: disagreement with institutional } \\
\text { protocol }{ }^{\mathrm{b}}(2)\end{array}$ \\
\hline Voriconazole & 2 & 5 & 0 & N/A \\
\hline Caspofungin & 1 & 4 & 0 & N/A \\
\hline Itraconazole & 2 & 3 & 0 & N/A \\
\hline
\end{tabular}

Abbreviations: N/A not applicable

a The same prescription may not be optimal for more than one reason

${ }^{b}$ Disagreement with institutional protocol, when available

patient-days in newborns) $[12,13]$. Remarkably, AF use in neonates was even lower in our centre (5 patients, accounting for 7 prescriptions, 6\%), in relation to a minimum incidence of neonatal candidiasis $(0.6 \%)$ that precludes the use of universal AF prophylaxis in low birth weight neonates $[14,15]$.

Regarding AF indication, prophylaxis was the main reason for prescriptions (63.2\%), followed by empirical treatment $(25 \%)$ and targeted treatment (11\%). These findings are consistent with data from the study performed by Santiago-García et al. that included 56 children with cancer and also the arm of paediatric patients from the ARPEC study $[8,12]$. In the neonate arm, prophylaxis was the reason for $46 \%$ of prescriptions, while treatment of suspected candidaemia accounted for the other $44 \%$ [12].

In terms of the distribution of AF, LAMB was the most prescribed drug in our study, followed by posaconazole. At our hospital, low-dose LAMB is the drug of choice for AF prophylaxis in high-risk cancer patients (relapsed or refractory leukaemia, HSCT preengraftment phase) due to its effectiveness and safety profile as shown in a previous paper by our group [16]. LAMB is also used in critically ill paediatric patients due to its stability with the concomitant use of continuous renal replacement therapy, as opposed to azole elimination [17]. On the other hand, posaconazole is mainly indicated in the HSCT post-engraftment period and in some primary immunodeficiencies (PID) such as chronic granulomatous disease. These data was quite different from other studies in which fluconazole is the most commonly prescribed agent [7, 11-13]. Nonetheless, all these studies include a high proportion, or exclusively include, neonates in whom Candida spp. is the main and almost only fungal pathogen [7, 11, 12]. Conversely, for paediatric, particularly children with cancer, PID or HSCT (a majority in our cohort), filamentous fungi should be considered, and other AFs with a broader spectrum play a major role [2, 10, 18, 19].

Regarding AF prescription evaluations, a high proportion (89\%) of prescriptions were considered correct, similar to the study by Santiago-García et al., that described $93 \%$ of appropriate AF prescriptions in a paediatric cancer department [8]. In contrast, a multicentric study by Ferreras-Antolín et al. considered that only $34 \%$ of prescriptions were optimal, similar to another study focused on adult patients with hospital-acquired candidemia that found $47 \%$ of correct prescriptions [7, 20]. Remarkably, the level of non-optimal prescriptions was higher in patients receiving treatment $(12 / 75 ; 16 \%)$ compared with prophylaxis $(12 / 144,8 \%)$, probably due to the higher availability of institutional protocols aimed at prophylaxis. Protocols were available for 117/144 (81\%) prophylactic prescriptions and for 40/75 (53\%) treatment prescriptions.

Regarding the cause of incorrect prescription, the leading reason was the lack of indication (unnecessary) (14/ 24), followed by disagreement with current recommendations $(12 / 24)$, incorrect dose $(7 / 24)$, incorrect duration (7/24) and inappropriate choice of AF (2/24). Interestingly, the proportions were generally similar to Santiago- 
Garcia et al., that described the lack of indication as the main cause of non-optimal prescription (7/13), followed by incorrect dosage $(5 / 13)$ or incorrect route of administration $(1 / 13)[8]$.

Some incorrect prescriptions were related to drugs not approved for paediatric use at the time of study (such as anidulafungin or isavuconazole). Although these drugs have shown clinical benefits in adults, paediatric use should be carefully evaluated and restricted to cases with no approved alternative for children. Moreover, for improving prescription's quality, decisions regarding antifungals should be made by a multi-disciplinary team. In centres with persuasive ASP, following experts' advice is of utmost importance.

With regards to dose, the incorrect rate (3\%) was significantly lower than in a previous study by Lestner et al. that found an inadequate dose as the main cause of incorrect AF use in paediatrics, identifying $47 \%$ of cases with doses lower than recommended by international guidelines [12]. The existence of specific tools for electronic prescription and the daily revision by the Pharmacy Department have probably contributed to this high rate of adequate dosing.

The limitations of this study were its single-centre nature and its PPS format, which may have meant that some prescriptions were missed. Moreover, a 4-month period including 11 surveys may not be sufficiently representative of overall practice, as several periods of viral epidemics can produce fever without bacterial cause and thus lead to increased empirical AF use. However, the results of this study regarding $\mathrm{AF}$ use are similar to annual AF consumption (DOT/100 PD) in our hospital (unpublished data). In 2018, most AF usage occurred in standard hospitalization patients, followed by PICU patients (63 and 34\%, respectively). In our cohort, these patients accounted for 70 and $24 \%$ of prescriptions, respectively. By therapeutic group, the AFs used most often in 2018 were azoles (45\% DOT/100 PD), LAMB (32\% DOT/100 PD), and echinocandins (23\% DOT/100 $\mathrm{PD})$. In terms of AF distribution in this PROAFUNGI study; LAMB was the most prescribed drug (46.2\%), followed by azoles (37\%) and echinocandins (16.7\%).

\section{Conclusions}

A high rate of optimal AF evaluation was globally demonstrated for all areas of a tertiary children's hospital, in relation to a well-established ASP that performs weekly audits and agreed protocols. However, this study identified specific areas of improvement, most of them related to the lack of an institutional protocol. These findings will guide the next actions of the AFS team, which will focus mainly on critical patients and the use of nonapproved drugs in children.

\section{Abbreviations}

AF: Antifungal drugs; AFS: Antifungal stewardship programmes; ARPE C: Antibiotic Resistance and Prescribing in European Children; ECMO: Extracorporeal membrane oxygenation; EORTC/MSG: European Organization for Research and Treatment of Cancer/Mycoses Study Group; HSCT: Hematopoietic stem cell transplantation; IFI: Invasive fungal infection; LAMB: Liposomal amphotericin B; mPPS: modified point-prevalence survey; NICU: Neonatal ICU; PICU: Paediatric ICU; PID: Primary immunodeficiencies; SOT: Solid organ transplantation; VAD: Ventricular assist device

\section{Acknowledgements}

We thank Helen Casas for English language support.

\section{Authors' contributions}

PSP and AFP concepted and designed the study. NMP and BGP collected and analysed the patient data. NMP, BGP, PSP and AFP interpreted the data and wrote the manuscript. SM, MTM and BR also contributed to the data evaluation and interpretation. All authors read and approved the submitted version and have agreed both to be personally accountable for the author's own contributions and to ensure that questions related to the accuracy or integrity of any part of the work are appropriately investigated, resolved, and the resolution documented in the literature.

\section{Funding}

This study was supported by an Investigator-Sponsored Research grant from Gilead Sciences. Gilead provided grant support for this study, but did not take part in the study design, data collection, or analysis of the results.

\section{Availability of data and materials}

The datasets used and/or analysed during the current study are available from the corresponding author on reasonable request.

\section{Ethics approval and consent to participate}

The Research Ethics Committee with Medicines from Vall d'Hebron Research Institute (Barcelona, Spain) approved the study in March 2018 (code VALANT-2017-01). The referred committee granted permission to access and analyse the study data. Patients' data was anonymised before its use. Study participants consent to participate is not applicable.

\section{Consent for publication}

Not Applicable.

\section{Competing interests}

The authors declare that they have no competing interests.

\section{Author details}

${ }^{1}$ Pediatric Infectious Diseases and Inmunodeficiencies Unit, Hospital Universitari Vall d'Hebron, Institut de Recerca Vall d'Hebron, Universitat Autònoma de Barcelona, Passeig de la Vall d'Hebron, 119-129, 08035 Barcelona, Spain. ${ }^{2}$ Pharmacy Department, Hospital Universitari Vall d'Hebron, Institut de Recerca Vall d'Hebron, Barcelona, Spain. ${ }^{3}$ Microbiology Department, Hospital Universitari Vall d'Hebron, Institut de Recerca Vall d'Hebron, Universitat Autònoma de Barcelona, Barcelona, Spain.

Received: 6 October 2020 Accepted: 6 January 2021

Published online: 22 January 2021

\section{References}

1. Hamdy RF, Zaoutis TE, Seo SK. Antifungal stewardship considerations for adults and pediatrics. Virulence. 2017:8:658-72. https://doi.org/10.1080/ 21505594.2016.1226721

2. Ramos JT, Francisco L, Daoud Z. Infección fúngica invasora en niños: diferencias y homologías con el adulto [Invasive fungal infections in children: similarities and differences with adults]. Rev Esp Quimioter. 2016;29 Suppl 1:59-65 Spanish. PMID: 27608317.

3. Science $M$, Timberlake K. Antifungal stewardship: a budding branch of antimicrobial stewardship. Pediatr Blood Cancer. 2020;67(4):e28145. https:// doi.org/10.1002/pbc.28145.

4. Barlam TF, Cosgrove SE, Abbo LM, et al. Implementing an antibiotic stewardship program: guidelines by the Infectious Diseases Society of 
America and the Society for Healthcare Epidemiology of America. Clin Infect Dis. 2016;62(10):e51-77. https://doi.org/10.1093/cid/ciw118.

5. Rodríguez-Baño J, Paño-Pardo JR, Alvarez-Rocha $L$, et al. Programs for optimizing the use of antibiotics (PROA) in Spanish hospitals: GEIH-SEIMC, SEFH and SEMPSPH consensus document. Enferm Infecc Microbiol Clin. 2012;30(1):22.e1-22.e23. https://doi.org/10.1016/j.eimc.2011.09.018.

6. Bienvenu AL, Argaud L, Aubrun F, et al. A systematic review of interventions and performance measures for antifungal stewardship programmes. J Antimicrob Chemother. 2018;73(2):297-305. https://doi.org/10.1093/jac/dkx388.

7. Ferreras-Antolín L, Irwin A, Atra A, et al. Neonatal antifungal consumption is dominated by prophylactic use; outcomes from the pediatric antifungal stewardship: optimizing antifungal prescription study. Pediatr Infect Dis J. 2019;38(12):1219-23. https://doi.org/10.1097/NF.0000000000002463.

8. Santiago-García B, Rincón-López EM, Ponce Salas B, et al. Effect of an intervention to improve the prescription of antifungals in pediatric hematology-oncology. Pediatr Blood Cancer. 2020;67(4):e27963. https://doi. org/10.1002/pbc.27963.

9. De Pauw B, Walsh TJ, Donnelly JP, et al. Revised definitions of invasive fungal disease from the European Organization for Research and Treatment of Cancer/invasive infections cooperative group. Clin Infect Dis. 2008;46(12): 1813-21. https://doi.org/10.1086/588660 Revised.

10. Rodríguez-Baño J, Paño-Pardo JR, Alvarez-Rocha L, et al. Programs for optimizing the use of antibiotics (PROA) in Spanish hospitals: GEIH-SEIMC, SEFH and SEMPSPH consensus document. Farm Hosp. 2012;36(1):33.e1-30 https://doi.org/10.1016/j.farma.2011.10.001.

11. Prasad PA, Coffin SE, Leckerman KH, et al. Pediatric antifungal utilization: new drugs, new trends. Pediatr Infect Dis J. 2008;27(12):1083-8. https://doi. org/10.1097/INF.0b013e31817eeee5.

12. Lestner $J M$, Versporten $A$, Doerholt $K$, et al. Systemic antifungal prescribing in neonates and children: outcomes from the antibiotic resistance and prescribing in European children (ARPEC) study. Antimicrob Agents Chemother. 2015;59(2):782-9. https://doi.org/10.1128/AAC.04109-14.

13. Stultz JS, Kohinke R, Pakyz AL. Variability in antifungal utilization among neonatal, pediatric, and adult inpatients in academic medical centers throughout the United States of America. BMC Infect Dis. 2018;18(1):1-9. https://doi.org/10.1186/s12879-018-3410-4.

14. Healy CM, Baker CJ. Fluconazole prophylaxis in the neonatal intensive care unit. Pediatr Infect Dis J. 2009;28(1):49-51. https://doi.org/10.1097/INF. Ob013e31819693ac.

15. Pappas PG, Kauffman CA, Andes DR, et al. Executive summary: clinical practice guideline for the Management of Candidiasis: 2016 update by the Infectious Diseases Society of America. Clin Infect Dis. 2016;62(4):409-17. https://doi.org/10.1093/cid/civ1194.

16. Mendoza-Palomar N, Soques E, Benitez-Carabante Ml, et al. Low-dose liposomal amphotericin B for antifungal prophylaxis in paediatric allogeneic haematopoietic stem cell transplantation. J Antimicrob Chemother. 2020; 75(8):2264-71. https://doi.org/10.1093/jac/dkaa149.

17. Bellmann R, Smuszkiewicz P. Pharmacokinetics of antifungal drugs: practical implications for optimized treatment of patients. Infection. 2017;45(6):73779. https://doi.org/10.1007/s15010-017-1042-z.

18. Steinbach WJ. Pediatric aspergillosis: disease and treatment differences in children. Pediatr Infect Dis J. 2005;24(4):358-64. https://doi.org/10.1097/01. inf.0000157218.37603.84

19. Mondain V, Lieutier F, Hasseine L, et al. A 6-year antifungal stewardship programme in a teaching hospital. Infection. 2013;41(3):621-8. https://doi. org/10.1007/s15010-013-0431-1.

20. Martín-Gutiérrez G, Peñalva G, Ruiz-Pérez de Pipaón M, et al. Efficacy and safety of a comprehensive educational antimicrobial stewardship program focused on antifungal use. J Inf Secur. 2020;80(3):342-9. https://doi.org/10. 1016/j.jinf.2020.01.002.

\section{Publisher's Note}

Springer Nature remains neutral with regard to jurisdictional claims in published maps and institutional affiliations.

Ready to submit your research? Choose BMC and benefit from:

- fast, convenient online submission

- thorough peer review by experienced researchers in your field

- rapid publication on acceptance

- support for research data, including large and complex data types

- gold Open Access which fosters wider collaboration and increased citations

- maximum visibility for your research: over $100 \mathrm{M}$ website views per year

At BMC, research is always in progress.

Learn more biomedcentral.com/submissions 